\title{
Schülerinnen und Schüler als Subjekte und Akteure von Schule
}

\author{
Ferdinand Eder (D) Angelika Paseka
}

Eingegangen: 30. April 2021 / Überarbeitet: 30. April 2021 / Angenommen: 4. Mai 2021 / Online publiziert: 8. Juni 2021

(C) Der/die Autor(en) 2021

Zusammenfassung In der Schul- und Bildungsforschung ist in den letzten Jahrzehnten der Blick auf das konkrete Erleben und Handeln der Kinder und Jugendlichen in ihrer Rolle als Schüler:innen und im Lebensraum Schule gegenüber der Fokussierung auf ihre fachlichen Leistungen in den Hintergrund getreten. Demgegenüber sieht der Zugang über die Subjekt- und Akteursperspektive die Schüler:innen als Akteure, die sich im Umfeld und unter dem Einfluss der Schule zu (selbst-)bewussten, denkenden und handelnden Individuen entwickeln und so „Subjektstatus“ erwerben.

Der Beitrag expliziert die begrifflichen Grundlagen dieses Zugangs, gibt einen kurzen Überblick über die darauf bezogene Entwicklung der Schule (und der Schulforschung) in den letzten Jahrzehnten, und umreißt die Konturen einer Bildungsforschung in der Akteurs- und Subjektperspektive. Abschließend werden die Beiträge des Schwerpunktheftes in diesen Forschungszugang eingeordnet.

Schlüsselwörter Schulforschung · Schülerforschung · Akteurskonzept · Schüler:innen als Akteure

Ferdinand Eder $(\varangle)$

Fachbereich Erziehungswissenschaft, Universität Salzburg, Salzburg, Österreich

E-Mail: Ferdinand.Eder@sbg.ac.at

Angelika Paseka

Fakultät für Erziehungswissenschaft, Universität Hamburg, Hamburg, Deutschland 


\section{Schoolchildren as subjects and actors in school}

Abstract In recent decades, the focus of educational research on the concrete experiences and actions of children and young people in their role as students in schools has faded into the background, compared to the measuring of competencies and achievement.

In contrast, a subject's/actor's paradigm directs the focus on the students as actors who grow up to (self-) conscious, thinking and acting individuals in the environment and under the influence of the school, and thus acquire "subject status".

The article defines the conceptual basis of this approach, gives a brief overview of the trends and developments of schools (and school research) over the past decades with respect to the status of students, and outlines the contours of student-related research from the perspective of actors and subjects. Finally, the contributions of the special issue are classified in this research access.

Keywords Educational research $\cdot$ Student research $\cdot$ Actors paradigm $\cdot$ Students as actors

In der Schul- und Bildungsforschung ist in den letzten Jahrzehnten der Blick auf das konkrete Erleben und Handeln der Kinder und Jugendlichen in ihrer Rolle als Schüler:innen und im Lebensraum Schule gegenüber der Fokussierung auf den fachlichen und leistungsbezogenen „output“ in den Hintergrund getreten. Dieser Trend steht in deutlichem Kontrast zur Ausrichtung der Forschung über Lehrpersonen. Hier stehen Entwicklung von Professionalität und professionelles Handeln, damit also auch Arbeitsprozesse, im Zentrum. Schüler:innen, so könnte man resümieren, werden eher an ihren „Erträgen“, Lehrer:innen an ihren (beruflichen, professionellen) Handlungen gemessen. Die hier sichtbar werdende Divergenz lässt sich mit dem Akteurskonzept in Verbindung bringen: Lehrpersonen werden eher als „Akteure“, Schüler:innen eher als (mehr oder weniger willige) „Adressaten“ von Bildungsprozessen verstanden.

\section{Begriffliche Abgrenzungen}

Die im Titel dieses Beitrags angeführten Leitbegriffe haben programmatischen Charakter: Das „Akteurs“-Konzept geht grundsätzlich davon aus, dass die Akteure von Schule, insbesondere auch die Schüler:innen, als handelnde Subjekte gesehen werden können, die sich aktiv mit ihrer Umwelt und den gegebenen Anforderungen auseinandersetzen. Dies lenkt das Augenmerk einerseits auf Regelmäßigkeiten (Gewohnheiten und Routinen) des Handelns, die ,,alltäglich ablaufenden Handlungsstrategien, Verstehensleistungen und Interaktionsprozesse der Akteure“" (Fend 2009, S. 121), andererseits auch darauf, wie sie mit ihnen unbekannten oder neuen Situationen umgehen, z.B. wenn sie erstmals mit dem System Schule konfrontiert werden. 
Im Blickfeld steht, wie Schüler:innen innerhalb eines bestehenden institutionalisierten Rahmens ihre Rollen und Aufgaben erleben, interpretieren und gestalten, aber auch ihre Verstricktheit mit den strukturierenden Rahmenbedingungen und das dem Handeln zugrunde liegende praktische Wissen.

Nach Hurrelmann (1993) entwickeln Individuen sich zu einer sozial handlungsfähigen Persönlichkeit ,,in Abhängigkeit von und in Auseinandersetzung mit den sozialen und den dinglich-materiellen Lebensbedingungen [...], die zu einem bestimmten Zeitpunkt der historischen Entwicklung einer Gesellschaft existieren“ (Hurrelmann 1993, S. 14). Das heißt, die Heranwachsenden werden als (Mit)-Produzent:innen von Wirklichkeit gesehen, die sie in ihren sozialen Praktiken hervorbringen. Schüler:innen greifen beim Versuch sich zu positionieren und eine eigene Persönlichkeit zu entwickeln, rekursiv auf vorhandene Strukturen zurück, (re)produzieren diese und können dabei deren Absichten unterlaufen (Giddens 1997, S. 77; Willis 2017).

Der „Subjekt“-Begriff schreibt Schüler:innen die Möglichkeit zu, sich im Umfeld und unter dem Einfluss der Schule zu (selbst-)bewussten, denkenden und handelnden Individuen zu entwickeln und so „Subjektstatus“ zu erwerben. Insofern ist der Subjektbegriff direkt mit dem Bildungsbegriff assoziiert: Der Prozess der Subjektwerdung entspricht weitgehend der Vorstellung von Bildung als einem Prozess des Mündigwerdens (Klafki 1985). Dieser Prozess setzt einen individuell gestaltbaren und veränderbaren institutionellen Rahmen als Bedingung seiner Möglichkeit voraus, und stößt damit rasch an die Grenzen von Schulsystemen, die auf die inhaltliche Standardisierung von Bildungsverläufen und Bildungsprozessen sowie auf Konditionierung des sozialen Verhaltens ausgerichtet sind. Beide Zugänge - Akteurskonzept und Subjektbegriff - implizieren jedenfalls, dass die Schüler:innen als Bezugspunkt für das Geschehen in der Schule verstanden werden.

In der Forschung lassen sich zwei Stränge ausmachen, Schüler:innen als Akteure und Subjekte einzubringen:

1. Erfassung der schulischen Umwelt im weitesten Sinne aus der Perspektive von Schüler:innen. Die Leitfrage lautet: Wie lassen sich die auf Schule bezogenen Kognitionen (beliefs), Interpretationen und Deutungsmuster erfassen, mit dem Ziel, die Handlungen der Schüler:innen bzw. ihre Entwicklung verstehen bzw. ,erklären“ zu können? Das betrifft z. B. ihre Einstellungen zum Lernen und den Umgang mit den Lernaufgaben, die Nutzung der Schule als Raum für soziale Interaktionen, die Bedeutung, die sie schulischen Wissensangeboten für die persönliche Entwicklung zuschreiben, oder den Einfluss von Schule auf aktuelles Befinden oder ihre überdauernde Lebensqualität. Dieser Zugang steht in der Tradition von Lewin (1963), Berger und Luckmann (1993 [1966]) und anderen, Schule als subjektiv wahrgenommene „Lebenswelt“ von Schüler:innen zu rekonstruieren.

2. Erfassung der Subjektwerdung durch Blick auf die Herstellungsprozesse, z. B. im Rahmen von ethnographischen Studien bzw. in Interviews und Gruppendiskussionen, die mit rekonstruktiven Verfahren ausgewertet werden. Die Leitfrage lautet hier: Was wird im Handeln (re)produziert und (re)konstruiert? Wie werden Kinder und Jugendliche adressiert und welche Inszenierungsformen nutzen sie selbst, um sich zu positionieren, z. B. als Mädchen bzw. Junge, als Schüler bzw. Schülerin (z.B. Paseka 2007)? Mit Blick auf die Praktiken: An welchen Normen und 
Common Sense-Theorien orientieren sie sich im Handeln bzw. im Reden über ihr Handeln? Dabei geht es auch um Fragen der Macht, die in Adressierungsprozessen genutzt und hergestellt wird (Butler 2018).

\section{Zur historischen Genese der Schüler:innenperspektive}

Die Teilnahme an internationalen Leistungsmessungen wie TIMSS und PISA führte spätestens seit Anfang dieses Jahrhunderts in Bildungspolitik und Bildungsforschung zu einer Zentrierung der Schulwahrnehmung auf die fachlichen Leistungen der Schüler:innen und die Rahmenbedingungen und Determinanten, unter denen sie erbracht werden. Diese „Outputorientierung“- forciert durch Botschaften der OECD, dass die Teilhabe an der weltweiten Wissensgesellschaft eine solche Intensivierung des schulischen Outputs erfordere, um die einzelnen Länder wettbewerbsfähig zu halten führte zu einer Fokussierung auf wenige Dimensionen (Leistungen in Mathematik, Lesen und Naturwissenschaften), die hinsichtlich der persönlichen Entwicklung und der gesellschaftlichen Teilhabe für basal gehalten werden, und zur Erfassung von dafür funktionalen sozialen und persönlichen Merkmalen, wie familiäre Herkunft oder Selbstwirksamkeit. Schülerleistungen werden verstanden als Resultate eines schulischen Produktionsmodells, das durch evidenzbasierte Forschung in seiner Effizienz optimiert werden kann.

Dieses Verständnis als „Produktionsmodell“ schließt an heterogene Entwicklungsphasen des Selbstverständnisses von Schule an: Die Schule der 1960er-Jahre - nach Schelsky eine „Zuteilungsapparatur von Lebenschancen“ (Schelsky 1965, S. 17) - sah ihre Aufgabe nicht zuletzt darin, einen (selbsterteilten) gesellschaftlichen Selektionsauftrag umzusetzen, und realisierte dies mit disziplinären Mitteln, die sie vor allem in der Wahrnehmung leistungsschwächerer Schüler:innen häufig als angstbesetzte Schreckensanstalt erscheinen ließ. Im Kontext der gesellschaftlichen Veränderungen seit den 1968er-Jahren beginnt dann eine nachhaltige Transformation in eine demokratisch organisierte und ,human“ ausgerichtete Institution, die darauf abzielte, jungen Menschen einen positiven Lern- und Lebensraum bereitzustellen, in dem Sach-, Selbst- und Sozialkompetenz vermittelt und gefördert werden sollten, nicht zuletzt gestützt durch die volkswirtschaftliche „Notwendigkeit“", durch „Ausschöpfung der Begabungsreserven“ dem rasant ansteigenden Bedarf an höherer Bildung zu entsprechen. Forschungen zum Schul- und Klassenklima (Fend 1977; Eder 1996), zum schülerorientierten Unterricht (Härle und Einsiedler 1976) u. Ä. versuchten, diese Transformation in eine „humane Schule“ zu unterstützen und diese als einen entwicklungsförderlichen Lebensraum zu konzipieren. ${ }^{1}$ Reduktion von Schulangst, Umsetzung respektvoller und reversibler Umgangsformen, partizipative Unterrichtsformen und Integration von Kindern und Jugendlichen mit Benachteiligungen charakterisierten dieses neue Verständnis von Schule. Förderung zielte stärker darauf ab, Kindern und Jugendlichen ,zur eigenen Lebensführung zu verhelfen“ (Bokelmann 1970, S. 185), ungeachtet der Selbstverständlichkeit, dass dabei der Erwerb von Wissen und Können eine zentrale Rolle spielt.

\footnotetext{
1 Unter diesem Aspekt erfolgte z. B. die Gründung der „Laborschule Bielefeld“ durch Hartmut von Hentig.
} 
Mit der für Deutschland und Österreich ernüchternden Einsicht aus den ersten internationalen Vergleichsuntersuchungen, hinsichtlich der Schulleistungen bestenfalls im Mittelfeld der OECD-Länder zu liegen, wurde diese stärker am Lernen und Leben der einzelnen Schüler:innen orientierte Perspektive durch eine Interventionslogik ersetzt, die darauf abzielte, durch flächendeckende Programme, z. B. zur Leseförderung, eine Steigerung der Leistungen zu erreichen. Die Schüler:innen als Subjekte und Akteure von Lernprozessen verschwanden weitgehend aus dem Blickfeld bzw. wurden zu Adressaten von Programmen. Selbst wenn Lernstrategien oder Problemlösungskompetenzen erfasst wurden, geschah dies primär in funktionaler Perspektive, inwieweit durch deren Optimierung bessere Leistungen erzielbar wären. Die Vorstellung einer ,humanen“ Schule wurde abgelöst durch das Konzept der „wirksamen Schule“ (beginnend mit Aurin 1990), und selbst die bildnerischen Fächer suchten mit der Idee eines „Kultur-PISA“ Anschluss an das neue Paradigma ${ }^{2}$.

Die deutschsprachige Bildungsforschung erlebt in dieser Phase eine zweite „empirische Wende", insbesondere durch die in Deutschland großzügige Förderung empirischer Bildungsforschung sowie durch die breite Umsetzung von Evaluation im Bildungsbereich. Der Großteil dieser Zugänge ist charakterisiert durch einen objektivierenden Blick ,von außen“, mit dem Ziel, jene Faktoren ausfindig zu machen, die für die geringe Wirksamkeit des Bildungssystems ursächlich sein könnten bzw. eine Steigerung seiner Wirksamkeit zur Folge hätten, wobei relativ unhinterfragt Wirksamkeit auf (fachliche) Leistung reduziert wird.

\section{Konturen einer Schüler:innenforschung in der Subjekt- und Akteursperspektive}

Schüler:innenforschung in einer Subjekt- und Akteursperspektive lässt sich - ausgehend von dem eingangs formulierten Begriffsverständnis - durch die folgenden Akzentuierungen umschreiben:

- Sie legt das Erkenntnisinteresse auf das (selbstgesteuerte) Handeln der Akteure, weniger auf dessen Ergebnis.

- Sie versucht, den subjektiv wahrgenommenen Handlungsraum der Subjekte und Akteure (,Lebenswelt“ sensu Lewin) zu erfassen und zu beschreiben.

- Sie sucht nach Zugängen, die an der unmittelbaren Verbesserung der Lebensverhältnisse der Betroffenen ansetzen.

- Sie bedient sich dazu Methoden, die eine authentische Wahrnehmung der Subjekte und Akteure ermöglichen; häufig sind qualitative Zugänge die Methode der Wahl.

Mit Blick auf die aktuelle Forschungslandschaft haben sich in diesem Rahmen sehr heterogene Zugänge entwickelt, die hier mit erheblichen Überschneidungen der verwendeten Kategorien dargestellt werden:

1. Arbeiten zu begrifflichen, konzeptuellen und methodologischen Aspekten (z.B. Tillmann 1980; Kelle und Breidenstein 1996; Rucker 2014; Velten et al. 2018)

\footnotetext{
2 Vgl. die Tagung „Evaluation kultureller Bildung?“ 13.-15.05.2007 in Wildbad-Kreuth.
} 
2. Forschungen zum „Erleben von Schule“. Dieser Zugang richtet sich darauf, wie verschiedene Aspekte von Schule von den „Betroffenen“ (Schüler:innen) erfahren und erlebt werden. Dazu zählen beispielsweise Untersuchungen

- zum Klima in der Schule und zur Qualität der erlebten sozialen Beziehungen zu Mitschüler:innen oder Lehrpersonen (Fend 1977; Arbinger und von Saldern 1984; Eder 1996),

- zum Befinden in der Schule allgemein, speziell auch von spezifischen Gruppen (Schüler:innen mit Behinderungen; mit Migrationshintergrund) (Eder 1995, 2007; Hascher 2004; Hascher und Hagenauer 2011),

- zum Ausmaß potenziell beeinträchtigender oder schädigender Emotionen wie Schul- und Prüfungsangst (Matischek-Jauk et al. 2018),

- zum Erleben von Schule und Unterricht (Bohnsack 2013; Peterlini 2016),

- zum Erleben von Partizipation (Eder 1998; Schmidt 2002; Bohnsack 2013; Wetzelhüter et al. 2013),

- zum Umgang der Schüler:innen mit Etikettierungen, Disziplinierungen, Beschämungen oder Strafen (Bohnsack 2013).

3. Rekonstruktion des impliziten und praktischen Wissens, das den Schüler:innen in ihrer Rolle als Schüler:innen verfügbar ist (,Schülerjob“, Breidenstein 2006; Helsper 2015), deren Wahrnehmung von Schule als Bildungseinrichung und Entwicklung eines schulbezogenen Habitus von privilegierten und nicht-privilegierten Kindern (Jünger 2010), aber auch die Erfassung von Kognitionen (beliefs) zu schulbezogenen Themen, z. B. Vorstellungen der Schüler:innen von Bildung, zum Lernen, zur Partizipation u. Ä.

4. Erfassung von Handlungen und Handlungsmustern im schulischen Kontext, z. B. Gestaltung des Lernens, insbesondere von Lernstrategien, Strategien und Praktiken zur Bewältigung von Schule, Strategien zum sozialen Umgang (Eder 1987; Breidenstein 2006; Petersen und Asbrand 2013; Forster-Heinzer et al. 2020).

5. Diagnose und Förderung der Erreichung normativer Erwartungen bezüglich Entwicklung des „Selbst“; Übernahme von Verantwortung für die eigene Entwicklung u. $\ddot{\text { A. }}$

6. Didaktischer Umgang mit Maßnahmen zur Förderung der Subjektwerdung, z. B.

- Mitsprache, Mitbestimmung in Schule und Unterricht (Rieker et al. 2016)

- Unterrichtsmethoden mit vermehrter Einbeziehung von Schüler:innen, z.B. „,agile Didaktik“, Forschendes Lernen, kooperative Settings im Unterricht, selbstreguliertes Lernen (Konrad und Traub 2011)

Wenig wissen wir noch darüber, wie Schüler:innen selbst ihre Bildungsentwicklung erleben und gestalten, also z.B. über den konkreten Stellenwert schulischen Wissens und schulischer Erfahrungen für ihr gegenwärtiges Leben. Wie wirkt sich die praktisch tägliche Konfrontation mit (subjektiv) neuem Wissen auf Lebensverständnis und Lebensführung aus? Welche Veränderungen im Denken, Fühlen und Handeln resultieren aus der kognitiven Auseinandersetzung mit Schulstoff? Welche Möglichkeiten finden Schüler:innen in Schule und Unterricht im Sinne der Subjekt-Entwicklung vor? Inwieweit hängen Wissens- und Wissenschaftsfeindlichkeit mit Erfahrungen in der Schule zusammen? Es erscheint fraglich, ob plakative Aussagen über den geringen Stellenwert schulischen Wissens für das tägliche Leben 
von Schüler:innen (im Vergleich etwa zur Bedeutung sozialer Kontakte) in dieser Allgemeinheit wirklich zutreffend sind.

In methodischer Hinsicht impliziert der Blick auf bzw. die Erforschung einer solchen Subjektperspektive häufig qualitative Zugänge. In zahlreichen Untersuchungen finden sich folglich narrative Zugänge, Interviews, Gruppendiskussionen, Vignettendarstellungen oder auch ethnographische Beobachtungen, jeweils begründet durch die Absicht, Zugänge zum Subjektverständnis von Kindern und Jugendlichen zu finden und deren Entwicklung einer eigenen Identität nachzuzeichnen. Diese Zugänge zielen darauf ab, Muster zu erkennen, das Typische herauszuarbeiten und Typologien zu erstellen. Es geht darum, nicht nur das Abfragbare, also das explizit zugängliche Wissen zu erfassen, sondern auch das implizite, stillschweigende, praktische Wissen der Akteure.

Verallgemeinerbare Aussagen, wie Schule von den Schüler:innen erlebt wird und welche Wirkung sie auf ihre Entwicklung hat, sind nur spärlich zugänglich. Fend und Berger (2016) haben auf Basis von Vergleichen für den Zeitraum von etwa 1983 bis 2013 gezeigt, dass die Schule ,humaner“ geworden ist. Man könnte von ,einer größeren Subjektorientierung in den pädagogischen Beziehungen in Schule“ (ebd., S. 882) sprechen: „Die pädagogische Kultur scheint sich in den letzten Jahrzehnten dramatisch in Richtung von mehr Empathie und Verständnis, hin zu einer stärkeren Reversibilität der Beziehungen und in Richtung einer Orientierung an den Bedürfnissen und Möglichkeiten der Kinder und Jugendlichen verändert zu haben“ (ebd., S. 883). Befürchtungen, diese stärker beziehungsorientierte Kultur würde erkauft mit Disziplinverlusten und Rückgängen im Leistungsbereich, hätten sich - so Fend und Berger - nicht bewahrheitet.

Längsschnittliche Studien wie diese können als Hinweise auf nachhaltige Veränderungsprozesse verstanden werden, die einer Absicherung durch weitere Forschung bedürfen. Dem stehen teilweise dramatische Einschätzungen der Alltagsrealität gegenüber: „Vorfälle von Mobbing, grausamer Unterricht, die tagtäglichen Verletzungen von Rechten der Schülerinnen und Schüler, ein unfaires Bildungssystem, unrealistischer Unterrichtsinhalt, der schlechte Umgang mit Kritik“, diagnostiziert der Frankfurter „StadtschülerInnenrat“, und fordert eine Verbesserung der Lage der Schüler:innen ${ }^{3}$.

\section{Ziel und Anlage des Schwerpunkthefts}

Die Art und Weise, wie Schüler:innen die Schule erleben und bewältigen, und welchen Einfluss sie auf ihre Entwicklung nehmen, hat zurzeit in der Schulforschung wenig Aufmerksamkeit gefunden und steht eher selten im Fokus von groß angelegten Forschungsstudien, verglichen etwa mit dem Ausmaß an Forschung, das sich mit den „klassischen“ Einflussfaktoren auf Leistungen, Verhalten oder Laufbahnen befasst. Bei diesen - Geschlecht, familiärer Hintergrund oder Migrationsstatus - handelt es sich um distale Merkmale, die nicht unmittelbar beeinflussbar sind, sondern ihre Wirkung in der Regel vermittelt über andere Einflussfaktoren ausüben. Die darauf

\footnotetext{
3 https://www.openpetition.de/petition/online/verbesserung-der-unterrichtsqualitaet.
} 
bezogene Forschung endet daher in der Regel mit dem Nachweis ihrer Effekte, verbunden mit Hinweisen auf allfällige Gegenmaßnahmen.

Bei den in der schüler:innenorientierten Perspektive angesprochenen Faktoren handelt es sich häufiger um proximale Merkmale, die das Handeln der Schüler:innen unmittelbar repräsentieren bzw. unmittelbar darauf Einfluss nehmen. Lern- und Bewältigungsstrategien im Umgang mit schulischen Aufgaben oder Situationen sind beispielsweise unmittelbar verhaltensrelevant und könnten durch entsprechende pädagogische Maßnahmen oder Interventionsprogramme auch modifiziert werden. Dies würde allerdings eine ,Fortbildung für Schüler:innen ' erfordern. Eine solche Forderung kann einerseits im Sinne eines ,Empowerment‘ für Kinder und Jugendliche gedacht werden und damit als eine Möglichkeit, Bildungsprozesse und die Entwicklung von Autonomie rechtzeitig anzubahnen. Andererseits könnte eine solche Forderung aber auch als ,Zurichtung ' und antizipierende Disziplinierung in die schulischen und gesellschaftlichen Normen kritisiert werden.

Ziel des vorliegenden Schwerpunktheftes ist es angesichts der hier aufgezeigten Befunde, den Blick stärker und unmittelbar(er) auf die konkrete Lebens- und Arbeitssituation der Schüler:innen zu richten, Routinen und Mechanismen im schulischen Umgang mit den Schüler:innen sichtbar zu machen, um zu einem besseren Verständnis ihrer Lage beizutragen, aufzuzeigen, wie schulische Situationen und Impulse verarbeitet und in das persönliche Leben integriert werden und dadurch begreifbar zu machen, ob und wie „Bildung“ stattfindet oder eher eine Zurichtung entlang bestehender Normen. Insgesamt steht dahinter die Frage, wie Schule konstituiert sein müsste, um den Ansprüchen zu genügen, die sich aus einer Theorie der Bildung und aus den bestehenden rechtlichen Vorgaben, beispielsweise der Konvention über die Rechte der Kinder, ableiten lassen.

Die aufgenommenen Beiträge resultieren aus einer Ausschreibung unter dem Titel „Schülerinnen und Schüler als Subjekte und Akteure von Schule“ und können als zwar selektive, aber doch ungesteuerte Auswahl aus der laufenden Forschung zu dieser Thematik verstanden werden. Die ursprünglich 40 Einreichungen beziehen sich auf begriffliche und methodologische Themen, Forschungen zum ,Erleben von Schule“, Erfassung von Handlungen und Handlungsmustern im schulischen Kontext, Erfassung von Kognitionen (beliefs) zu schulbezogenen Ereignissen, aber auch von implizitem Wissen, das dem Handeln der Subjekte, hier: der Kinder und Jugendlichen, in schulischen Kontexten zugrunde liegt, Entwicklung von Identität sowie (didaktische) Maßnahmen, die als Förderung der „Subjekt“-Werdung verstanden werden können. Dementsprechend bringt das Heft Analysen und Forschungen

1. zum Subjekt- und Rechtsstatus von Kindern bzw. Schüler:innen, insbesondere zur Partizipation in der Schule

Thomas Rucker, Elmar Anhalt und Kira Ammann: Der Subjektstatus des Schülers/der Schülerin - Erziehungstheoretische Überlegungen

Daniela Müller-Kuhn, Pascale Herzig, Julia Häbig und Enikö Zala-Mezö: Student participation in everyday school life - Linking different perspectives

2. zur Entwicklung eines „Habitus“ von Schüler:innen zur Bewältigung von Schule Matthias Martens und Barbara Asbrand: „Schülerjob“ revisited: Zur Passung von Lehr- und Lernhabitus im Unterricht 
Aytüre Türkyilmaz „,Wenn ich lerne, dann werde ich auch Erfolg haben später“ Kinder als Akteure in Prozessen struktureller (Re-)Produktion

3. zum Erleben und zu den Auswirkungen sozialer Beziehungen

Gerda Hagenauer, Christina Wallner-Paschon und Clara Kuhn: Austrian students' experiences of supportive relationships with teachers, peers, and parents and the mediating effect of school belonging in the context of their academic and nonacademic outcomes

Sina Schürer, Stefanie van Ophuysen und Sophie Michalke: Der Zusammenhang von Schuleinstellung und Qualität kind-und klassenbezogener pädagogischer Beziehungen von Lehrkräften

4. zum Umgang mit Ungerechtigkeit, Diskriminierung und Etikettierungen in der Schule

Sophia Richter und Antje Langer: Im Namen der Gerechtigkeit? Ungerechtigkeitsthematisierungen von Schüler*innen als Positionierungen

Anja Steinbach und Anke Spies: Bildungsbiografische Rekonstruktionen. Erfahrungen von Schüler/innen mit verbalen und nonverbalen Anrufungen und Diskriminierungen

Norman Krause, Cindy Ballaschk, Friederike Schulze-Reichelt, Julia Kansok-Dusche, Sebastian Wachs, Wilfried Schubarth und Ludwig Bilz: „Ich lass mich da nicht klein machen! " Eine qualitative Studie zur Bewältigung von Hatespeech unter Jugendlichen in der Schule

Antje Gansewig und Maria Walsh: Die Sichtweise von Schülerinnen und Schülern auf eine primärpräventive Maßnahme eines ehemaligen Rechtsextremen: Befunde einer Evaluationsstudie und Empfehlungen für Bildungsakteure

5. und nicht zuletzt zum Umgang mit dem COVID19-verursachten Ausfall von Schule

Wassilios Baros, Ulrike Greiner, Mishela Ivanova und Aida Delic: Perspektiven auf Schul- und Lebenswelten in Schüler/innen-Narrationen während der CoronaKrise im Frühjahr 2020

Die präsentierten Untersuchungen decken nur einen Teil des oben beschriebenen Zugangs ab, eröffnen aber Blicke in ein Forschungsfeld, in dem noch viele Fragen offen sind, insbesondere jene nach den Auswirkungen von Schule auf die individuelle personale Entwicklung, jenseits des Erwerbs von (fachlichen) „Kompetenzen“. Oder anders formuliert: ob und wie in der Schule „Bildung“ im Verständnis von Subjektwerdung erfolgt und die „Persönlichkeit“ der Schüler:innen entsteht.

Funding Open access funding provided by Paris Lodron University of Salzburg.

Open Access Dieser Artikel wird unter der Creative Commons Namensnennung 4.0 International Lizenz veröffentlicht, welche die Nutzung, Vervielfältigung, Bearbeitung, Verbreitung und Wiedergabe in jeglichem Medium und Format erlaubt, sofern Sie den/die ursprünglichen Autor(en) und die Quelle ordnungsgemäß nennen, einen Link zur Creative Commons Lizenz beifügen und angeben, ob Änderungen vorgenommen wurden.

Die in diesem Artikel enthaltenen Bilder und sonstiges Drittmaterial unterliegen ebenfalls der genannten Creative Commons Lizenz, sofern sich aus der Abbildungslegende nichts anderes ergibt. Sofern das betreffende Material nicht unter der genannten Creative Commons Lizenz steht und die betreffende Handlung 
nicht nach gesetzlichen Vorschriften erlaubt ist, ist für die oben aufgeführten Weiterverwendungen des Materials die Einwilligung des jeweiligen Rechteinhabers einzuholen.

Weitere Details zur Lizenz entnehmen Sie bitte der Lizenzinformation auf http://creativecommons.org/ licenses/by/4.0/deed.de.

\section{Literatur}

Arbinger, R., \& von Saldern, M. (1984). Schulische Umwelt und soziales Klima in Schulklassen. Psychologie in Erziehung und Unterricht, 31, 81-99.

Aurin, K. (Hrsg.). (1990). Gute Schulen - Worauf beruht ihre Wirksamkeit? Bad Heilbrunn: Klinkhardt.

Berger, P.L., \& Luckmann, T. (1993). Die gesellschaftliche Konstruktion der Wirklichkeit (10. Aufl.). Frankfurt am Main: Fischer.

Bohnsack, F. (2013). Wie Schüler die Schule erleben. Zur Bedeutung der Anerkennung, der Bestätigung und der Akzeptanz von Schwäche. Opladen, Berlin, Toronto: Barbara Budrich. http://site.ebrary.com/ lib/alltitles/docDetail.action?docID=10642005

Bokelmann, H. (1970). Pädagogik: Erziehung, Erziehungswissenschaft. In J. Speck \& G. Wehle (Hrsg.), Handbuch pädagogischer Grundbegriffe (S. 178-267). München: Kösel.

Breidenstein, G. (2006). Teilnahme am Unterricht. Ethnographische Studien zum Schülerjob (1. Aufl.). Studien zu Schul- und Bildungsforschung, Bd. 24. Wiesbaden: VS, GWV. https://doi.org/10.1007/ 978-3-531-90308-8.

Butler, J. (2018). Kritik der ethischen Gewalt. Frankfurt am Main: Suhrkamp.

Eder, F. (1987). Schulische Umwelt und Strategien zur Bewältigung von Schule. Psychologie in Erziehung und Unterricht, 34, 100-110.

Eder, F. (1995). Das Befinden von Schülerinnen und Schülern in den öffentlichen Schulen - Ergebnisse der Repräsentativerhebung. In: F. Eder (Hsg.): Das Befinden von Kindern und Jugendlichen in der Schule. (S. 24-168). Innsbruck: StudienVerlag.

Eder, F. (1996). Schul- und Klassenklima. Ausprägung, Determinanten und Wirkungen des Klimas an weiterführenden Schulen. Innsbruck: StudienVerlag.

Eder, F. (1998). Schule und Demokratie. Untersuchungen zum Stand der demokratischen Alltagskultur an Schulen. Innsbruck: StudienVerlag.

Eder, F. (2007). Das Befinden von Kindern und Jugendlichen in der österreichischen Schule. Befragung 2005. Innsbruck: StudienVerlag.

Fend, H. (1977). Schulklima. Weinheim: Beltz.

Fend, H. (2009). Neue Theorie der Schule. Einführung in das Verstehen von Bildungssystemen. Bd. 2. Wiesbaden: VS, GWV. https://doi.org/10.1007/978-3-531-91788-7.

Fend, H., \& Berger, F. (2016). Ist die Schule humaner geworden? Sozialhistorischer Wandel der pädagogischen Kulturen in Schule und Familie in den letzten 30 Jahren im Spiegel der LifE-Studie. Zeitschrift für Pädagogik, 62(6), 861-885.

Forster-Heinzer, S., Nagel, A., Biedermann, H., \& Reichenbach, R. (2020). Impression Management im Unterricht: Über die Entwicklung eines Instruments zur Erfassung von Selbstpräsentationsstrategien. Zeitschrift für Bildungsforschung, 10(2), 147-173. https://doi.org/10.1007/s35834-020-00273-4.

Giddens, A. (1997). Die Konstitution der Gesellschaft. Grundzüge einer Theorie der Strukturierung (3. Aufl.). Theorie und Gesellschaft, Bd. 1. Frankfurt/Main: Campus. Original erschienen 1977

Härle, H., \& Einsiedler, W. (Hrsg.). (1976). Schülerorientierter Unterricht. Donauwörth: Auer.

Hascher, T. (Hrsg.). (2004). Schule positiv erleben. Erkenntnisse und Ergebnisse zum Wohlbefinden von Schülerinnen und Schülern. Bern: Haupt.

Hascher, T., \& Hagenauer, G. (2011). Schulisches Wohlbefinden im Jugendalter - Verläufe und Einflussfaktoren. In A. Ittel, H. Merkens \& L. Stecher (Hrsg.), Jahrbuch Jugendforschung (Bd. 31, S. 15-45). Wiesbaden: VS. https://doi.org/10.1007/978-3-531-93116-6_1.

Helsper, W. (2015). Schülerbiographie und Schülerhabitus. In S. Sandring, W. Helsper \& H.-H. Krüger (Hrsg.), Jugend (Bd. 5, S. 131-159). Wiesbaden: Springer. https://doi.org/10.1007/978-3-65803543-3_6.

Hurrelmann, K. (1993). Einführung in die Sozialisationstheorie. Über den Zusammenhang von Sozialstruktur und Persönlichkeit (4. Aufl.). Weinheim, Basel: Beltz.

Jünger, R. (2010). Schule aus der Sicht von Kindern. In A. Brake \& H. Bremer (Hrsg.), Alltagswelt Schule. Die soziale Herstellung schulischer Wirklichkeiten (S. 159-183). Weinheim, München: Juventa. 
Kelle, H., \& Breidenstein, G. (1996). Kinder als Akteure: Ethnographische Ansätze in der Kindheitsforschung. Zeitschrift für Sozialisationsforschung und Erziehungssoziologie, 16(1), 49-65.

Klafki, W. (1985). Konturen eines neuen Allgemeinbildungskonzepts. In W. Klafki (Hrsg.), Neue Studien zur Bildungstheorie und Didaktik (S. 12-30). Weinheim und Basel: Beltz.

Konrad, K., \& Traub, S. (2011). Selbstgesteuertes Lernen. Grundwissen und Tipps für die Praxis (3. Aufl.). Baltmannsweiler: Schneider-Verl. Hohengehren. http://www.socialnet.de/rezensionen/isbn.php? isbn=978-3-8340-0516-8

Lewin, K. (1963). Feldtheorie in den Sozialwissenschaften. Bern: Huber.

Matischek-Jauk, M., Krammer, G., \& Reicher, H. (2018). The life-skills program Lions Quest in Austrian schools: implementation and outcomes. Health Promotion International, 33(6), 1022-1032. https:// doi.org/10.1093/heapro/dax050.

Paseka, A. (2007). Geschlecht lernen am Schauplatz Schule. SWS-Rundschau, 47(1), 51-72.

Peterlini, H. K. (2016). Fenster zum Lernen. Forschungserfahrungen im Unterrichtsgeschehen - Einführung und Einblicke in die Suche nach einem neuen Verständnis von Lernen. In S. Baur \& H.-K. Peterlini (Hrsg.), An der Seite des Lernens. Erfahrungsprotokolle aus dem Unterricht an Südtiroler Schulen - ein Forschungsbericht (S. 21-29). Innsbruck: StudienVerlag.

Petersen, D., \& Asbrand, B. (2013). Anpassungsleistungen von Schülerinnen und Schülern beim Übergang von der Grundschule in die weiterführenden Schulen. Zeitschrift für Qualitative Forschung, 14(1), 49-65. https://doi.org/10.3224/zqf.v14i1.15452.

Rieker, P., Mörgen, R., Schnitzer, A., \& Stroezel, H. (2016). Partizipation von Kindern und Jugendlichen. Formen, Bedingungen sowie Möglichkeiten der Mitwirkung und Mitbestimmung in der Schweiz (1. Aufl.). Kinder, Kindheiten und Kindheitsforschung, Bd. 15. Wiesbaden: Springer VS. https://doi. org/10.1007/978-3-658-10991-2.

Rucker, T. (2014). Erkenntnisfortschritt durch Problematisierung, oder: Über das Verhältnis von ,Bildung ‘ und ,Subjektivation‘. Zeitschrift für Pädagogik, 60(2), 908-2014.

Schelsky, H. (Hrsg.). (1965). Schule und Erziehung in der industriellen Gesellschaft. Würzburg: Werkbund.

Schmidt, R. (2002). Schülerpartizipation im Schulleben und Unterricht. In H.-H. Krüger, S. Reinhardt, C. Kötters-König, N. Pfaff, R. Schmidt, A. Krappidel \& al (Hrsg.), Jugend und Demokratie - Politische Bildung auf dem Prüfstand (S. 103-114). Wiesbaden: VS. https://doi.org/10.1007/978-3-66307863-0_4.

Tillmann, K. J. (1980). Das Schulsystem und seine sozio-psychischen Auswirkungen auf die Schüler. In W. Spiel (Hrsg.), Konsequenzen für die Pädagogik (1). Die Psychologie des 20. Jahrhunderts, (Bd. 11, S. 617-633). Göttingen: Hogrefe.

Velten, K., Alexi, S., \& Höke, J. (2018). Die Erfassung von Kinderperspektiven und ihr Potenzial für Forschung und Professionalisierung. In S. Miller, B. Holler-Nowitzki, B. Kottmann, S. Lesemann, B. Letmathe-Henkel \& N. Meyer, et al. (Hrsg.), Profession und Disziplin. Grundschulpädagogik im Diskurs. Jahrbuch Grundschulforschung, (Bd. 22, S. 127-137). Wiesbaden: Springer VS.

Wetzelhüter, D., Paseka, A., \& Bacher, J. (2013). Partizipation in der Organisation Schule aus der Perspektive der Schülerinnen und Schüler. In S. M. Weber, M. Göhlich, A. Schröer, C. Fahrenwald \& H. Macha (Hrsg.), Organisation und Partizipation. Beiträge der Kommission Organisationspädagogik. Organisation und Pädagogik, (Bd. 13, S. 157-168). Wiesbaden: Springer.

Willis, P.E. (2017). Learning to labor. How working-class kids get working-class jobs (Legacy editions). New York: Columbia University Press. Original erschienen 1977 